\title{
Modeling and scientific application of scintillation results
}

\author{
E. J. Fremouw and J. A. Secan
}

Physical Dynamics, Inc.

(Received April 15, 1983; revised August 22, 1983; accepted September 22, 1983.)

\begin{abstract}
Efforts continue to update and improve empirical models of the mean morphological behavior of ionospherically produced scintillation. While often intended for engineering application, these models also can be scientifically useful to the extent that they describe not only the signal fluctuations but also the plasma density irregularities that cause them. Such a model is contained in the computer code called WBMOD, which employs the phase screen propagation theory to calculate the power law spectral index and strength (at a fluctuation frequency of $1 \mathrm{~Hz}$ ) of phase scintillation and the standard deviation of phase, for a user-specified system. Explicit provision is made for an ionospheric outer scale, but the relevant phase spectrum is cut off by a system parameter in most applications. The intensity scintillation index $S_{4}$ is calculated from weak-scintillation theory and corrected for the saturating effect of multiple scatter on the basis of Rice statistics. The irregularity model in WBMOD describes threedimensionally anisotropic (sheetlike) irregularities on the nightside of the high-latitude scintillation zone and axially symmetric (rodlike) irregularities elsewhere. The current status of WBMOD, which is undergoing continued refinement, is described in this paper for all latitude regimes. Plans for future development of its irregularity model are stated, and it is suggested that such models should now move in the direction of incorporating irregularity dynamics.
\end{abstract}

\section{INTRODUCTION}

Given the many observations that have been made of ionospherically imposed radio wave scintillation at numerous locations around the world, there is need for an orderly means to assemble the data. Such a scientifically useful repository of information is represented by computer-based models intended for application to transionospheric communication systems. This paper is an attempt to illustrate such models, by means of a particular one, namely that developed over a period of several years by one of the authors (E.J.F.) and several co-workers and now residing in a computer code called WBMOD.

The objectives of scintillation modeling for scientific purposes are by no means in conflict with those for engineering application, but the emphasis is somewhat different. First and foremost, for scientific purposes, we expect a model to provide a description of ionospheric irregularities and not only of the signal scintillations they produce. Furthermore, if the model is itself based on scintillation data rather than, say, on in situ measurements or observations of spread $F$, we expect it to contain information on

\section{Copyright 1984 by the American Geophysical Union.}

Paper number 351556.

0048-6604/84/003S-1556\$08.00 irregularity shape as well as strength. Such a capability is the forte of the scintillation technique.

The description may be static, but parameterization to describe change is necessary if the model is to yield insights about irregularity development. For this purpose, changes in both shape and strength should be described, including changes that depend on location over the earth and that cycle with time, on duration scales ranging from diurnal, through seasonal, to that of solar epoch. Greater scientific utility, still, will be realized if description is included of changes related to solar-geophysical disturbance events.

\section{ELEMENTS OF A SCIENTIFICALLY USEFUL MODEL}

Scattering theory. To meet the foregoing objectives, it is necessary to separate the geometrydependent propagation aspects of the scintillation problem from the geophysical aspects. In WBMOD this is accomplished by means of the phase screen formulation of Rino [1979], the most pertinent results of which are contained in (1) through (5), below:

$$
\begin{gathered}
p \approx q+1 \\
T=N(q) \lambda^{2}\left(C_{s} L\right) \sec \theta G V_{e}^{q}
\end{gathered}
$$

where $p$ and $T$ are the power law spectral index and 
power spectral density (the latter at a fluctuation frequency of $1 \mathrm{~Hz}$ ) of the phase scintillation spectrum, and where $G(a, b, \delta)$ is a geometrical enhancement factor and $V_{e}\left(V_{s}, V_{d}, a, b, \delta\right)$ is the effective scan speed across contours of plasma density, while $\lambda$ is the radio wavelength and $N$ is a normalization factor. Other quantities on the right-hand side of (1) and (2) are defined in this and the next subsection.

The phase variance, a convenient phase scintillation index, is the integral of the spectrum over the system or processing window, which typically is cut off at a low frequency $f_{c}$ such that

$$
f_{c} \gg f_{0}=\frac{V_{e}}{2 \pi \alpha}
$$

where $\alpha$ is the outer scale of ionospheric structure. That is, the phase variance is given by

$$
\sigma_{\phi}^{2}=2 \int_{f_{c}}^{\infty} \frac{T d f_{f}}{\left(f_{0}^{2}+f_{f}^{2}\right)} \approx \frac{2 T}{(p-1) f_{c}^{(p-1)}} \approx \frac{2 T}{q f_{c}^{q}}
$$

where the first approximation follows from (3) and the second stems from ignoring diffraction effects on phase in accord with (1), where $q$ is the onedimensional spectral index in situ.

The corresponding intensity scintillation index, which is the normalized (by the mean squared) intensity variance, is related to the phase perturbation and free space diffraction, in the absence of multiple scatter, by

$$
S_{4 w}^{2}=\frac{M(q)}{N(q)} T \frac{F}{G} \frac{z^{q / 2}}{V_{e}^{q}}
$$

where $F(q, a, b, \delta)$ is the Fresnel filter factor, $Z(\lambda, h)$ is the Fresnel zone size, and the ratio $M / N$ adjusts the normalization. In the absence of strong focusing, the saturation of $S_{4}$ in the multiple scatter regime can be accounted for, in accordance with Rice statistics [Fremouw and Rino, 1976], by

$$
S_{4}^{2} \approx 1-\exp \left(-S_{4 w}^{2}\right)
$$

Equations (1) through (5) are based squarely on a power law spatial spectrum for the irregularities. They are appropriate, therefore, for modeling a majority of ionospheric structures. By the same token, they would have to be reformulated to permit modeling of some potentially important minorities, such as multiple-regime power laws [Rino et al., 1981; Basu et al., 1983; Fremouw et al., 1983a].

Irregularity parameters. The foregoing equations also are based on the geometrical formulation of Singleton [1970] and therefore afford a fairly general description of three-dimensionally anisotropic irregularities. On the safe assumption that one elongation axis of anisotropic irregularities is aligned along the magnetic field, such irregularities are specified by two axial ratios, $a$ (along the field) and $b$ (across the field), and an orientation angle $\delta$ (defined in relation to the local magnetic $L$ shell).

The (static) geometrical enhancement factor depends on the shape of the irregularities through $a, b$, and $\delta$, and the (dynamic) effective scan speed depends not only on that shape but also on the line-of-sight scan velocity $V_{s}$ and the (plasma) irregularity drift velocity $\mathbf{V}_{d}$. Both these geometrically dependent factors affect the strength of scatter as well as does the height-integrated strength $C_{s} L$ of the spatial spectrum.

Perusing the right-hand sides of (1), (2), and (5) discloses three irregularity shape parameters, $a, b$, and $\delta$; one irregularity strength parameter, $C_{s} L$; and two additional ionospheric parameters, $h$ and $V_{d}$, that dictate the three readily available scintillation observables, $p, T$ (alternatively, $\sigma_{\phi}$ ), and $S_{4}$; clearly, the set of irregularity parameters is underdetermined.

\section{MODELING APPROACH}

The upshot of the foregoing discussion is that one needs either to use some inductive approach to irregularity parameterization, such as iterative modeling, or to enlist the aid of some other source of information. In development of WBMOD, we have in fact done both. Primarily, WBMOD is based on data from the DNA Wideband Satellite (hence the name). Because Wideband was a low orbiter, data from it permit some geometry-based modeling of irregularity shape. By the same token, it is difficult to sort out geophysical from geometrical effects as the satellite scanned across latitude circles and through a range of pertinent propagation angles.

To date, our basic approach has been to assume that shape-dependent geometrical factors are relatively unchanging from pass to pass as compared with the geophysical variability of irregularity strength. Under this assumption, some parameters have been fixed from external sources.

The layer height is placed essentially at the middle of the $F$ layer, $350 \mathrm{~km}$ except near the equator. Originally, this value came from spaced receiver measurements at a mid-latitude station by McClure [1964]. More recently, we have noticed that it is very near the $F$ layer peak at auroral latitudes as well, as indicated by the Chatanika radar. The layer is placed at 
$500 \mathrm{~km}$ altitude near the magnetic equator, since the plume region seems to extend from below to well above that height under strong scintillation conditions.

We take the large-scale drift velocity of the irregularities, $\mathbf{V}_{d}$, to be that of the bulk plasma, and for high latitudes we are in the process of incorporating in WBMOD the convective electric field model recently published by Heelis et al. [1982]. At equatorial latitudes we have used the extensive incoherent-scatter observations from Jicamarca [Fejer et al., 1981] to model a diurnally cyclic variation, with eastward drifts reaching $100 \mathrm{~m} / \mathrm{s}$ at 2200 local time, westward drifts reaching $50 \mathrm{~m} / \mathrm{s}$ at 1000 , and reversals taking place just before 1600 and just after 0400. A magnetic field model is central to the geometrical coordinate system, and we are using International Geomagnetic Reference Field (IGRF) '80.

Three parameters currently are set as constants in accord with the average values indicated by the Wideband data base. First, on the basis of (1) and a representative value of 2.5 for the observed phase spectral index $p$, we have fixed the in situ index $q$ at 1.5. There is an interesting systematic departure from constancy in the value of $p$ in the auroral zone [Fremouw et al., 1983a] and indications of a systematic trend in $q$ at equatorial latitudes [Livingston et al., 1981], but these behaviors will not be incorporated in the model until more extensive documentation and understanding have been accumulated.

The Wideband data base is consistent with an outer scale in all regions that is larger than the spatial-spectrum cutoff imposed by data processing, which is at least $4 \mathrm{~km}$ for the line-of-sight average produced in scintillation measurements. Thus we employ the large outer-scale approximation. We also take all irregularities to be elongated along the magnetic field and, in the case of the only known threedimensionally anisotropic ones, along the local $L$ shell, which is indicated by setting the orientation angle $\delta$ to zero.

The most difficult parameters to establish have turned out to be the along-field and cross-field axial ratios, $a$ and $b$, which quantify the anisotropy. There seems to be no evidence for cross-field anisotropy in scintillation-producing irregularities near the equator, so we have set $b$ to unity there. We take the field-aligned axial ratio $a$ to be very large (30) there, in accord with long-standing and ambitious interferometer measurements by Koster et al. [1966].

In the auroral region we have put in considerable effort to establish aggregate values for $a$ and $b$. It is quite clear from Wideband that daytime irregularities near Fairbanks usually are axially symmetric in the daytime and sheetlike at night [Fremouw and Lansinger, 1981]. We have modeled $b$, therefore, as a diurnal variable and have attempted to set its nighttime value and the auroral-latitude value for $a$ from the width of the observed geometrical enhancement region. This has been fairly successful, but it leaves some inconsistency with the observations, as we will soon see.

The difficulty with setting $a$ and $b$ from geometrical enhancement of the scattering strength is that irregularity strength changes fairly sharply with latitude in the auroral region. We intend, therefore, to set the latitudinal dependence of the nighttime axial ratios on the basis of interferometer observations recently analyzed by Livingston et al. [1982]. This will allow us to refine the layer height, if necessary, on the basis of the ratio of intensity to phase scintillation indices and then to proceed with modeling the main geophysical variable by means of an iterative calculation unencumbered with the need for axial-ratio iteration.

As always, the main iterative loop will be closed before a final test against reserved data sets.

\section{CURRENT MODEL}

Using the foregoing doubly iterative procedure, we have arrived at the following shape and strength model.

$$
\begin{gathered}
q=1.5 \quad \delta=0 \\
a=30-11\left(1+\operatorname{erf} \frac{\lambda_{m}-20^{\circ}}{3^{\circ}}\right) \\
b=1+0.75\left[1+\cos \frac{\pi\left(t_{m}-2\right)}{12}\right]\left[1+\operatorname{erf} \frac{\lambda_{m}-\lambda_{b}}{\lambda_{k}}\right] \\
\left(C_{s} L\right)^{1 / 2}=H\left(\lambda_{m}, t_{m}, \bar{R}, K p\right)+M\left(\lambda_{m}, t\right) \\
+E\left(\lambda_{m}, \lambda_{g}, d, t, D, \bar{R}\right) \quad\left(\mathrm{el}^{2} / \mathrm{m}^{2}\right)^{1 / 2}
\end{gathered}
$$

where

$\lambda_{m}$ geomagnetic latitude;

$t_{\mathrm{m}}$ geomagnetic time;

$\overline{\boldsymbol{R}}$ smoothed Zurich sunspot number;

$K p$ planetary geomagnetic activity index;

$\lambda_{g}$ geographic latitude of the local field line apex;

$d$ magnetic declination at the field line apex;

$t$ time of day;

$D$ day of the year. 
In (8), $\lambda_{b}$ denotes the average invariant latitude of the high-latitude scintillation boundary, and $\lambda_{h}$ the standard deviation of its instantaneous latitude for a given time of day and $K p$ value. The average boundary latitude is modeled as a function of the latter two independent variables as follows:

$$
\lambda_{b}=71.8^{\circ}-1.5 K p-5.5 \cos \frac{\pi\left(t_{m}-2\right)}{12}
$$

Equation (7) describes very elongated, field-aligned irregularities within $20^{\circ}$ of the magnetic equator, the axial ratio of which decreases to 8 at middle and high latitudes. Equation (8) describes quasi-sheets with a cross-field axial ratio of 4 (half as large as the alongfield axial ratio) on the nightside of the earth poleward of the scintillation boundary but axially symmetric irregularities elsewhere.

The height-integrated spectral strength of the irregularities is modeled, in (9), as the sum of three terms, which respectively dominate at high, middle, and equatorial latitudes. The high-latitude term is as follows:

$$
H=4.3 \times 10^{11}(1+0.05 \bar{R})\left[1+\operatorname{err} \frac{\lambda_{m}-\lambda_{b}}{\lambda_{h}}\right]
$$

Equation (10) describes a scintillation boundary offset from the geomagnetic pole in the antisunward direction and rotated 2 hours from the noonmidnight meridian. It migrates equatorward with increasing magnetic disturbance at the rate of $1.5^{\circ}$ per $K p$ unit.

The mid-latitude term, $M\left(\lambda_{m}, t\right)$, is a very simple latitudinal and diurnal variable.

The equatorial term of the model currently is undergoing substantial revision. Based on preWideband and very early Wideband data, it describes a region of disturbance that peaks some $10^{\circ}$ on each side of the geomagnetic equator and an hour and a half before local midnight. Until recently, the seasonal variation included equinoctial peaking and a filling in of activity in the local summer season. This behavior is consistent with differences in seasonal patterns observed by Wideband at Kwajalein and Ancon [Livingston, 1980] but not with a similarity in MARISAT scintillation behavior observed at Natal and Accra [Aarons et al., 1980]. The latter two stations are in the southern and northern geographic hemispheres, respectively, but activity at both of them minimizes in the June solstice season.

The complicated seasonal variation of equatorial scintillation, differing as it does in different longitude sectors, long has been an enigma. Recently, R. T. Tsunoda (unpublished manuscript, 1983) has made a significant advance in resolving the enigma by postulating that scintillation-producing irregularities are most likely to develop in the equatorial $F$ layer when the integrated $E$ region Pedersen conductivity experiences its maximum longitudinal gradient. Such behavior would be consistent with the theoretical view that the convective instability ultimately leading to scintillation-producing irregularities affects an entire flux tube and not just localized plasma [Anderson and Haerendel, 1979; Zalesak et al., 1982].

On the basis of the foregoing postulate, Tsunoda predicted that scintillation activity would maximize at a given longitude at times of year when the solar terminator is most nearly aligned with the geomagnetic flux tubes in that sector. He then employed scintillation data from several equatorial and nearequatorial observing stations to test his hypothesis. He found scintillation maxima in seasons when the time difference between $E$ region sunset at opposite ends of the relevant field line minimizes, in accord with his prediction.

For modeling, we have parametrized the behavior reported by Tsunoda in terms of the angle at the dip equator between the solar terminator and the field line through an $F$ layer point of interest. Computation results are the two days of the year, $D_{1}$ and $D_{2}$ on which that angle, calculated for a field line passing through the location of interest at $350 \mathrm{~km}$ altitude, is zero (i.e., the two days on which the geographic azimuth angle of the terminator at the relevant point on the dip equator equals the magnetic declination there). A straightforward geometrical development yields the following two solutions:

$$
\begin{aligned}
& D_{1}=(365 / 2 \pi) \gamma_{0}+81.4 \\
& D_{2}=(365 / 2 \pi)\left(\pi-\gamma_{0}\right)+81.4
\end{aligned}
$$

where

$$
\gamma_{0}=\sin ^{-1}\left[\cos \lambda_{g} \sin d / \sin \varepsilon\right]
$$

and where $\lambda_{g}$ is the geographic latitude of the field line/dip equator crossing, $d$ is the magnetic declination there, and $\varepsilon$ is the ecliptic angle, $23.5^{\circ}$. The field line apex parameters, $\lambda_{g}$ and $d$, are determined by means of a field line trace.

Instead of calculating $D_{1}$ and $D_{2}$, one might seek to parameterize average irregularity strength directly on the magnetic azimuth of the terminator (i.e., the difference between its geographic azimuth and the magnetic declination at the field line apex). As yet, 
however, Tsunoda's hypothesis only identifies times of the year when field line instability is most likely. Translating that hypothesis, even when fully tested, into a seasonal/longitudinal dependence of average strength, in the absence of extensive statistical data and/or more refined physical insights, would constitute little more than an educated guess. We have chosen, therefore, to employ Tsunoda's hypothesis only to identify $D_{1}$ and $D_{2}$ as a basis for empirical modeling.

At present, we are using $D_{1}$ and $D_{2}$ to locate two seasonal Gaussian distributions, as follows:

$$
\begin{aligned}
E_{D} & =1.0+C_{D}\left\{\exp \left[-\left(\frac{D-D_{1}}{D_{0}}\right)^{2}\right]\right. \\
& \left.+\exp \left[-\left(\frac{D-D_{2}}{D_{0}}\right)^{2}\right]\right\}
\end{aligned}
$$

where $C_{D}$ is a seasonal-variation weighting factor to be determined empirically and $D_{0}$ is a seasonal Gaussian half width also to be so established. The half width will determine the degree of "filling in" between peak seasons, the season filled in most depending upon whether

$$
D_{2}-D_{1} \gtrless \frac{365}{2}
$$

Equation (14) then will be combined with the existing sunspot number, diurnal, and latitudinal variation of equatorial irregularity strength to produce

$$
\begin{aligned}
E= & C_{e} E_{D}(1+0.04 \bar{R}) \exp \left[-\frac{(t-22.5)^{2}}{t_{r}^{2}}\right] \\
& \cdot\left\{\exp \left[-\frac{\left(\lambda_{m}-10^{\circ}\right)^{2}}{\left(10^{\circ}\right)^{2}}\right]+\exp \left[-\frac{\left(\lambda_{m}+10^{\circ}\right)^{2}}{\left(10^{\circ}\right)^{2}}\right]\right\}
\end{aligned}
$$

where

$$
t_{r}=\begin{aligned}
& 3 \\
& 6
\end{aligned} \quad t \lessgtr 22.5
$$

and where the lead constant $C_{e}$ has yet to be accurately calibrated against Wideband data.

\section{RESULTS AND DISCUSSION}

As an example of model results, the solid curves in Figure 1 show the calculated standard deviation of phase for Wideband passes over Poker Flat, Alaska, for three different geometries and for low and moderate $K p$. The dotted curves show the average behavior of the indicated number of passes occurring under similar conditions. The calculations portray most of the trends and behaviors of the data base, but there are notable disagreements.

Since both the shape of the irregularities and the latitude dependence of their strength affect the shape of the curves, it appears that we have reached the limit of refinement from iterating the model against observed scintillation indices alone at Poker Flat. Fortunately, more direct estimates of anisotropy axial ratio are being made from the Wideband interferometer data [Livingston et al., 1982]. These measurements show a definite latitudinal decrease in axial ratio poleward of Poker Flat, with sheets and quasi-sheets reverting to decreasingly elongated rods. These results can provide guidance for specifying shape, so that scintillation index iterations can be used to establish the consistent latitudinal dependence of irregularity strength.

The result of Livingston et al. [1982] is part of an interesting research trend regarding sheetlike irregularities. Starting from acceptance of the notion that cross-field anisotropy is the norm in the high-latitude ionosphere, detailed analyses now have restricted that picture in both time of day and latitude. It now seems very likely that the region of sheetlike irregularities is coincident with the region of large-scale structures seen in the $F$ layer by means of the Chatanika radar [Muldrew and Vickrey, 1982]. These structures appear as total electron content (TEC) enhancements on low-resolution records of dispersive phase, such as those reported by Leitinger et al. [1982].

Of particular interest is the likely relationship between the large-scale plasma enhancements and the flux of soft electrons near the edge of the diffuse aurora. Figure 2 compares the quiet-time location of enhanced soft-electron fluxes observed by Gussenhoven et al. [1981] with that of the scintillation boundary described by (10). The two boundaries are rotated by the same amount from the noon-midnight meridian, and their centers are separated in a sense consistent with irregularity production by antisunward convection. Moreover, their equatorward expansion rates with increasing magnetic activity, indicated at the lower left of the figure, are essentially identical. It seems that such comparison between scintillation data summarized by means of modeling and other observations should provide some insight into irregularity dynamics.

More often, perhaps, it is disagreements between 

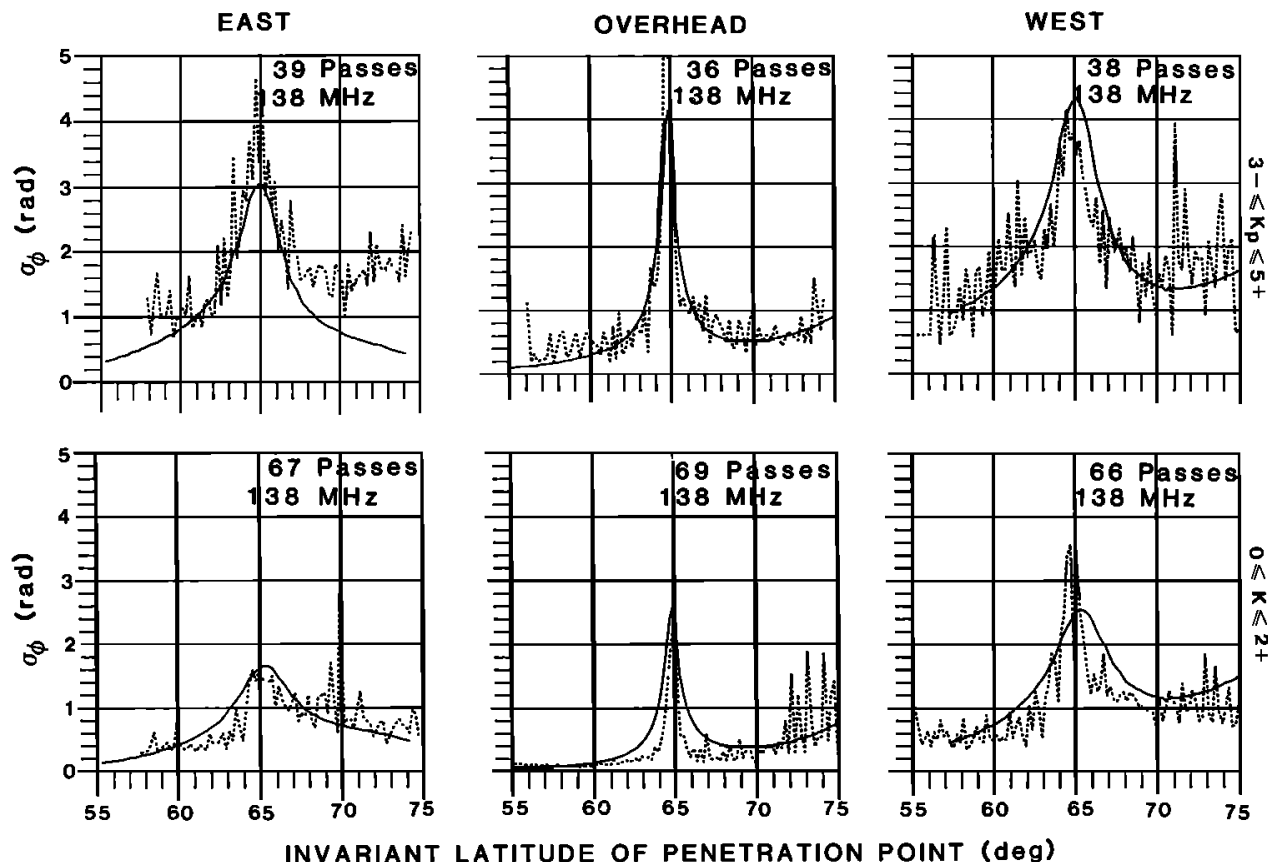

Fig. 1. Comparison of nighttime, VHF phase scintillation index calculated (solid) by means of Program WBMOD and observed (dotted) at Poker Flat, Alaska, in the Wideband Satellite Experiment. Left: premidnight passes to the east of the station. Center: near-midnight passes essentially along the geomagnetic meridian. Right: postmidnight passes to the west of the station. Bottom: $0 \leq K p \leq 2+$. Top: $3-\leq K p \leq 5+$. The number of passes in each data set is indicated in the upper right-hand corner of each grid. Calculated values are for a single representative pass.

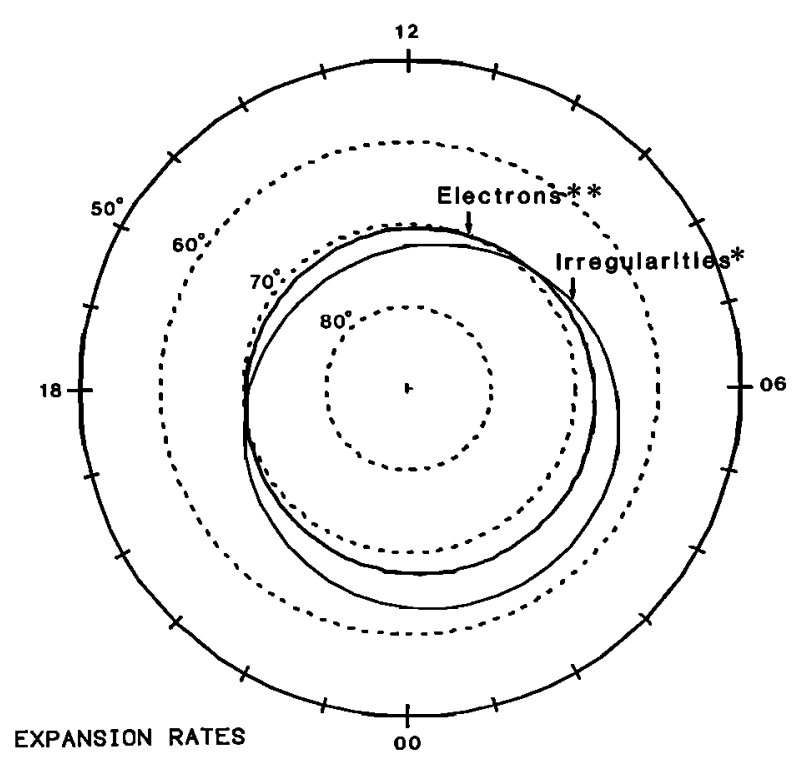

* $1.6^{\circ} / K_{P}$ TO $2.2^{\circ} / K p$

Fig. 2. Quiet-time boundaries, in invariant latitude and magnetic time, of modeled high-latitude irregularities and observed [Gussenoven et al., 1981] enhanced fluxes of soft electrons. Expansion rates for each with increasing geomagnetic activity are given at lower left. the current model and existing observations that provide research guidance. An example is given in Figure 3, which compares WBMOD calculations with the average phase scintillation index observed in 10 nighttime passes of Wideband over Poker Flat. Although based on very limited data, the figure nonetheless shows a sharp discrepancy between model and data between $55^{\circ}$ and $60^{\circ}$ invariant latitude. The observed scintillation enhancement in that region probably is located at and just equatorward of the plasmapause. This phenomenon has received attention from Houminer et al. [1981], and it will be explored further by means of the forthcoming HILAT satellite [Fremouw et al., 1983b]. Meanwhile, we intend to incorporate it descriptively into the mid-latitude term of the existing model in WBMOD.

At equatorial latitudes the current model has yet to be calibrated against the full population of relevant data from Wideband. We intend to do so soon, using as a guide the comparative morphology of intensity scintillation in the American and Pacific sectors published by Livingston [1980]. The main modeling task outstanding at equatorial latitudes is to extend the description of seasonal variation accurately to other longitudes, including provision for the 


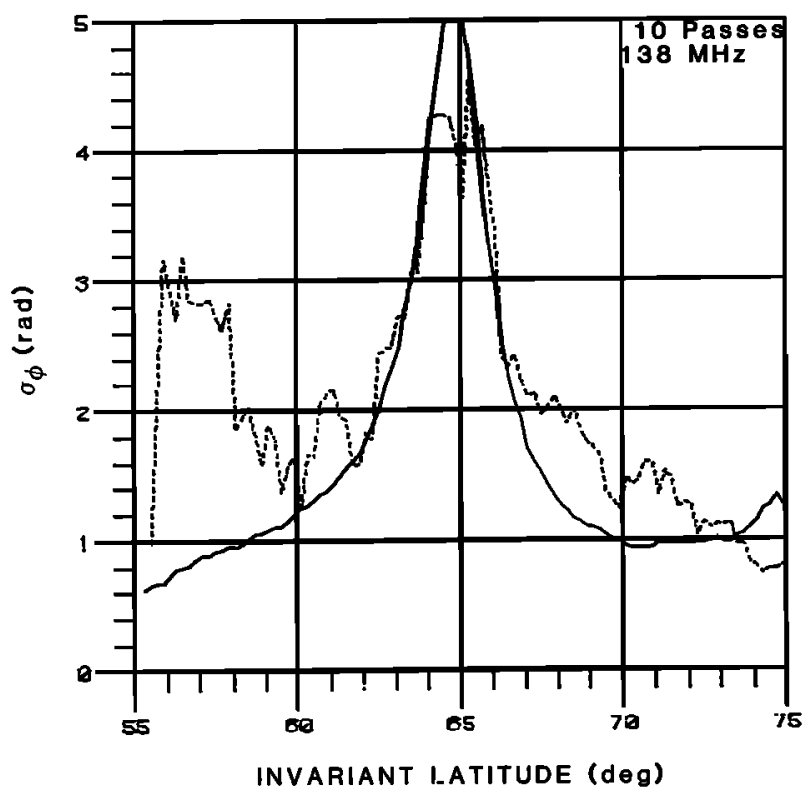

Fig. 3. Comparison of model results (solid) and observed values (dotted) of VHF phase scintillation index for geomagnetically very disturbed $(K p \geq 6-)$ nighttime conditions. Note sharp disagreement near latitude of plasmapause.

especially strong scintillation in the Atlantic sector. Organized data sets from equatorial stations, especially of phase scintillation and especially from the Indian and Asian sectors, stand as the greatest need for advancing descriptive modeling of scintillation.

\section{CONCLUSION}

The emphasis in this paper has been on the scientific aspects of scintillation modeling. Such models may continue to be called on more often for applications use, as they have been in the past. In this role they represent the repository of scintillaticn data and an end product of scintillation research. As in other fields, however, science and application continually stimulate one another. The need for more generally useful and robust applications-oriented computer codes necessitates modeling in terms of ionospheric parameters. This, in turn, provides a means for identifying shortfalls in the data base and physical understanding.

Scintillation modeling may be at a developmental threshold. While climatological description is by no means complete, modelers should soon be able to take on the challenge of incorporating some ionospheric dynamics into their work. At equatorial latitudes, for instance, our current efforts involve both empirical use of scintillation data and application of the theoretically based insight of R. T. Tsunoda (unpublished manuscript, 1983). At high latitudes the currently ongoing multiexperiment HILAT Satellite mission [Fremouw et al., 1983b] holds promise for modelers. Close comparison between scintillation data and measurements of plasma dynamical quantities should lead to qualitative advances in irregularity models.

Acknowledgment. Program WBMOD has been developed under sponsorship of the Defense Nuclear Agency under contract DNA001-81-C-0092.

\section{REFERENCES}

Aarons, J., J. P. Mullen, J. P. Koster, R. D. DaSilva, J. R. Medeiros, R. T. Medeiros, A. Bushby, J. Pantoja, J. Lanat, and M. R. Paulson, Seasonal and geomagnetic control of equatorial scintillations in two longitudinal sectors, J. Atmos. Terr. Phys., 42, 861-866, 1980.

Anderson, D. N., and G. Haerendel, The motion of depleted plasma regions in the equatorial ionosphere, $J$. Geophys. Res., 84(A8), 4251-4256, 1979.

Basu, S., S. Basu, J. P. McClure, W. B. Hanson, and H. E. Whitney, High resolution topside in situ data of electron densities and $\mathrm{VHF} / \mathrm{GHz}$ scintillations in the equatorial region, J. Geophys. Res., 88(A1), 403-415, 1983.

Fejer, B. G., D. T. Farley, C. A. Gonzales, R. F. Woodman, and C. Calderon, $F$ region east-west drifts at Jicamarca, $J$. Geophys. Res., 86, 215-218, 1981.

Fremouw, E. J., and J. M. Lansinger, Dominant configurations of scintillation-producing irregularities in the auroral zone, J. Geophys. Res., 86(A11), 10,087-10,093, 1981.

Fremouw, E. J., and C. L. Rino, Modeling of transionospheric radio propagation, Rep. $R A D C-T R-76-35$, Rome Air Dev. Center, Griffiss Air Force Base, N. Y., 1976.

Fremouw, E. J., J. A. Secan, and J. M. Lansinger, Spectral behavior of phase scintillation in the sub-auroral enhancement region, paper presented at Winter U.S. Meeting, Boulder, Colo., URSI, Jan. 5, 1983a.

Fremouw, E. J., et al., The HiLat program, Eos Trans. $A G U$, 64(18), 163-170, $1983 b$.

Gussenhoven, M. S., D. A. Hardy, and W. J. Burke, DMSP/F2 electron observations of equatorward auroral boundaries and their relationship to magnetospheric electric fields, J. Geophys. Res., 86(A2), 768-778, 1981.

Heelis, R. A., J. K. Lowell, and R. W. Spiro, A model of the height-latitude ionospheric convection pattern, J. Geophys. Res., 87(A8), 6339-6345, 1982.

Houminer, Z., J. Aarons, and F. Rich, Production and dynamics of high-latitude irregularities during magnetic storms, $J$. Geophys. Res., 86(A12), 9939-9944, 1981.

Koster, J. R., I. Katsriku, and M. Tete, Studies of the equatorial ionosphere using transmission from active satellites, Annu. Sum. Rep. 1, contract AF61(052)-800, Univ. of Ghana-Legon, Accra, Ghana, 1966

Leitinger, R., G. K. Hartmann, W. Debenhardt. A. Hedberg, and P. Tanskanen, The electron content of the ionosphere and the southern boundary of diffuse aurora, J. Atmos. Terr. Res., 44(4), $369-374,1982$. 
Livingston, R. C., Comparison of multifrequency equatorial scintillation: American and Pacific sectors, Radio Sci., 15(4), 801$814,1980$.

Livingston, R. C., C. L. Rino, J. P. McClure, and W. B. Hanson, Spectral characteristics of medium-scale equatorial $F$ region irregularities, J. Geophys. Res., 86(A4), 2421-2428, 1981.

Livingston, R. C., C. L. Rino, J. Owen, and R. T. Tsunoda, The anisotropy of high-latitude nighttime $F$-region irregularities, Top. Rep. 1, contract DNA001-81-C-0076, SRI International, Menlo Park, Calif., 1982.

McClure, J. P., The height of scintillation-producing ionospheric irregularities in temperate latitudes, J. Geophys. Res., 69(13), 2775-2780, 1964.

Muldrew, D. B., and J. F. Vickrey, High-latitude $F$ region irregularities observed simultaneously with ISIS 1 and the Chatanika radar, J. Geophys. Res., 87(A10), 8263-8272, 1982.
Rino, C. L., A power law phase screen model for ionospheric scintillation, 1, Weak scatter, Radio Sci., 14(6), 1135-1145, 1979.

Rino, C. L., R. T. Tsunoda, J. Petriceks, R. C. Livingston, M. C. Kelley, and K. D. Baker, Simultaneous rocket-borne beacon and in situ measurements of equatorial spread $F$-Intermediate wavelength results, J. Geophys. Res., 86(A4), 2411-2420, 1981.

Singleton, D. G., Dependence of satellite scintillations on zenith angle and azimuth, J. Atmos. Terr. Phys., 32, 789-803, 1970.

Zalesak, S. T., S. L. Ossakow, and P. K. Chaturvedi, Nonlinear equatorial spread $F$ : The effect of neutral winds and background Pedersen conductivity, J. Geophys. Res., 87(A1), 151$166,1982$.

E. J. Fremouw and J. A. Secan, Physical Dynamics, Inc., P. O. Box 3027, Bellevue, WA 98005. 